\title{
Pharmacological and Neuroprotective Natural Products in the Treatment of Alzheimer's Disease
}

\author{
A.Mary Prashanthi ${ }^{1}$, Vissarapu Naga Lakshmi ${ }^{2}$ \\ ${ }^{1}$ M.Pharmacy Student, Department of Pharmacology, ${ }^{2}$ M.Pharmacy Student, Department of Pharmaceutical \\ Chemistry, Hindu College of Pharmacy, Amaravathi Road, Guntur-522002.(A.P), India
}

Corresponding Author: A.Mary Prashanthi

\begin{abstract}
Alzheimer's illness (AD) is a progressive, neurodegenerative issue and has become an incredible medical condition especially among the old individuals, for the most part happens after the age of 55 years and expansion in frequency with propelling age, however its therapy alternatives are generally not many and inadequate. The reason for AD isn't actually known, yet is thought to incorporate both hereditary and natural elements (multifactorial). There is no known remedy for Alzheimer's sickness, since it is beyond the realm of imagination to expect to invert the demise of synapses however the indications can be overseen by some pharmacological specialists and Neuroprotective Natural Products. In spite of the improvement of various pharmacological methodologies throughout the years pointed toward treating Alzheimer's Disease (AD) without a doubt, not many have been supported for clinical use in patients. To date there still exists no illness adjusting treatment that could forestall or protect the intellectual debilitation, especially of memory aquisition, that is normal for $\mathrm{AD}$. One of the opportunities for this situation may be that most of medication disclosure endeavors centers around result proportions of diminished neuropathological biomarkers normal for $\mathrm{AD}$, without taking into acount neuronal cycles fundamental for the age and support of memory measures. In endeavors to find new methodologies for $\mathrm{AD}$ treatment, regular items have stirred interest in the examination local area and in the drug business for their neuroprotective movement, focusing on various obsessive instruments related with $\mathrm{AD}$. A wide assortment of normal items from various beginnings has been assessed preclinically and
\end{abstract}

clinically for their neuroprotective instruments in forestalling and lessening the multifactorial pathologies of $\mathrm{AD}$. It is accepted that normal item blends or concentrates containing different bioactive mixtures that can work additively or synergistically to display various neuroprotective systems may be a compelling methodology in $\mathrm{AD}$ drug discovery.

Keywords: Alzheimer's disease (AD); neurodegenerative disease, natural products, pharmacological agents.

\section{INTRODUCTION ${ }^{[1-3]}$}

Alzheimer's disease (AD), found by Dr. Alois Alzheimer in 1906, is presently the main ongoing neurodegenerative illness, influencing more than and around 50 million individuals overall. Age is viewed as the greatest danger factor for $\mathrm{AD}$. Being the most widely recognized reason for dementia among more seasoned grown-ups and recorded by the Centers for Disease Control and Prevention (CDC) as the 6th driving reason for death in the US, AD may really be positioned third, soon after coronary illness and malignant growth, in significant reasons for death for more established individuals. As a neurodegenerative infection, $\mathrm{AD}$ leisurely and irreversibly obliterates memory, cognizance, and at last the capacity to do every day exercises, prompting the requirement for full-time care, which is generally normal among individuals more than 65 , in spite of the fact that it happens in the more youthful population ${ }^{[4]}$. 
The specific natural reason for $\mathrm{AD}$ in a great many people is as yet not completely comprehended; the signs of this infection are accepted to be the unusual testimony of insoluble $\beta$-amyloid peptide $(\mathrm{A} \beta)$ and the amassing of neurofibrillary tangles (NFTs) of phosphorylated tau protein in neuronal cytoplasm, which brings about decay and neuron demise. A few organic cycles have been connected to these neurodegenerative changes including neuroinflammation, exhaustion or deficient union of synapses, oxidative pressure, breakdown of calcium homeostasis, and strange ubiquitination. Subsequently, neurons and neurotransmitters taking part in memory measures are harmed, prompting a decrease in learning capacity and other psychological capacities. Every one of the right now accessible drugs for $\mathrm{AD}$, with the exception of memantine which blocks NMDA receptors in the mind from abundance incitement that can harm nerve cells, increment the measure of synapses in the cerebrum to briefly work on psychological indications.

Alzheimer's illness (AD) is a problem portrayed by reformist disability of psychological capacities, especially memory. The old style neuropathological biomarkers that have been associated with $A D$ side effects incorporate the conglomeration of amyloid $\beta$ (A $\beta$ ) peptide into decrepit plaques and the hyperphosphorylation of tau protein that outcomes in neurofibrillary tangles. Extraordinary endeavors have been made to find a viable pharmacological technique to balance memory disability in $\mathrm{AD}$. To date just five medications have been endorsed for clinical use to treat the infection with restricted viability in long haul treatment. Such a low achievement rate recommends that tracking down a more powerful way to deal with treat AD is fundamental in order to stop or essentially postpone the infection common memory debilitation. Since the 1960s pre-clinical and clinical exploration has shown that memory relies upon the age and support of synchronized neuronal action that produces cerebral rhythms especially slowfrequency theta $(\theta)$ movement (8-12 $\mathrm{Hz})$ and quick recurrence gamma $(\gamma)$ action $(30-80 \mathrm{~Hz})$. Both $\theta$ and $\gamma$ rhythms are essentially adjusted in AD patient's minds in the later phases of the sickness. However total clinical and exploratory proof shows that these changes of $\theta$ and $\gamma$ rhythms are available effectively in the beginning phases of AD.

One significant theory dependent on exploratory proof proposes that $A \beta$ in its non-totaled structure could be answerable for the principal changes of $\theta$ and $\gamma$ rhythms, driving at last to memory interruption in people just as creature models. In spite of the significant job of $\theta$ and $\gamma$ rhythms for memory measures, pre-clinical examination growing new pharmacological techniques against $\mathrm{AD}$ is predominantly centered around the decrease of exemplary neurotic biomarkers like $\mathrm{A} \beta$, disregarding whether an effective decrease of $A \beta$ is additionally joined by a rebuilding of the mind rhythms fundamental for ordinary memory work. All in all a thin spotlight on result proportions of diminished neuropathological biomarkers normal for $\mathrm{AD}$, without considering neuronal cycles vital for the age and support of memory cycles, for example, useful biomarkers as perception significant neuronal organization motions probably prevents the disclosure of effective treatments. Subsequently, this survey sums up existing exploratory proof with respect to pharmacological methodologies advancing the improvement of $\theta$ and $\gamma$ rhythms in solid just as AD creature models.

Because of degeneration of cholinergic neurons the levels of the acetylcholine (ACh) synapse in the body gets low which prompts side effects of AD. Restraint of compound acetylcholinesterase (AChE) what breaks ACh to choline and acetic acid derivation, grouping of $\mathrm{ACh}$ expansions in neural connection. Exorbitant incitement of the NMDA receptor causes expansion in section of calcium particle on the receptor through particle direct outcomes in neuronal injury or demise. 
Advertisement the executives are accepted to be mind boggling since no specific treatment has been created to control its indications. Most of the medicines accessible for $\mathrm{AD}$ underline on treating neurological and conduct issues that can disintegrate as the sickness advances. Restricted adequacy, incidental effects, and helpless patient consistence of contemporary arrangement of medication warrant the investigation of elective restorative techniques of $\mathrm{AD}$. Alternatives from home grown and customary cures like conventional Indian medication (Ayurveda) and traditional Chinese medication (TCM) are being explored due to the normal methodology with immaterial incidental effects when contrasted with regular allopathy techniques.

These conventional frameworks have likewise added to the developing rundown of significant therapeutic spices, mixtures and one of kind administration techniques. From a mid 90's century natural medication is supposed to be the best treatment to fix any sickness. However, from most recent couple of years there is expansion in development of natural treatment in India just as in different nations. The expansion being used of home grown medication is because of its normal beginning and it have less incidental effects when contrasted with any remaining treatment. Treatment with home grown medication can likewise be called as Neutraceuticals approach. The term neutraceuticals was given by Stephen Defelice MD and is gotten from "sustenance" and "drug". Natural medication which are utilized in treatment are gotten from therapeutic plants, minerals and so on. Various home grown medication are utilized to treat neurodegenerative sicknesses like Alzheimer's Disease (AD). Home grown plants have different exercises like cancer prevention agent, calming, anticholinesterase, antiamyloid impact, $\beta$ and $\gamma$-secretase inhibitors and because of this load of exercises which are available in plants it assists with boosting memory, oversee social just as mental manifestations related with $\mathrm{AD}$.

These medications' adequacy fluctuates a ton separately and is restricted in term. Different methodologies, including focusing on $\mathrm{A} \beta$ plaques and tau NFT conglomerations decreasing oxidative pressure and neuroinflammation, and so on, have been endeavored, with various new medication applicants being assessed in preclinical and clinical examinations. Regular sources, including plants, creatures, organisms, and the marine world, furnish plentiful bioactive mixtures with complex constructions and novel pharmacological properties. As one of the significant wellsprings of medication revelation, regular items and their separated mixtures have been widely concentrated in endeavors to foster more successful medications for the administration of AD. Indeed, the cholinesterase inhibitor galantamine is a characteristic item itself and rivastigmine is a semi-engineered subordinate of a characteristic item called physostigmine.

Blends or concentrates of normal items may enjoy benefits contrasted with singular regular mixtures, as they have various synchronous objective methodologies, which could be a clever treatment alternative for $\mathrm{AD}$, considering the intricacy of its pathophysiology. Mounting proof has proposed that spices or home grown definitions, along with combinations got from other regular sources, may give psychological advantages to $\mathrm{AD}$ patients. Subsequently, different regular sources and their concentrates are broadly utilized in creature models and AD patients. The regular item concentrates or combinations that might have neuroprotective impacts through different instruments for the anticipation and treatment of $\mathrm{AD}$.

\subsection{Etiology ${ }^{[4,5]}$}

The specific reason in case AD isn't known yet it is accepted that few ecological and hereditary danger factors trigger the side effects of $\mathrm{AD}$. A portion of the causing 
factors are given beneath,Age-related changes in the mind, Researchers have asserted that more seasoned grown-ups have more danger of having $\mathrm{AD}$. Researchers are as yet realizing, how age-related changes in the mind might hurt neurons and add to Alzheimer's harm. Hereditary qualities, It is realized that qualities play a significant role in the advancement of AD. Beginning stage Alzheimer's is exceptionally uncommon structure, It happens in the middle of the age of 30 to 60 . Most instances of beginning stage Alzheimer's are familial Alzheimer's sickness, brought about by changes in one of three realized qualities acquired from a parent. A great many people with Alzheimer's infection have late-beginning Alzheimer's, which as a rule creates after age, 60. Natural/way of life factors, Conditions like coronary illness, stroke, hypertension, diabetes, and heftiness are additionally connected as hazard factors for AD.

\subsection{Symptoms ${ }^{[6,7]}$}

The manifestations which are seen in gentle, moderate and extreme type of AD are trouble in performing undertakings, learning new things, playing complex games, level disposition and character changes, mind flights, daydreams and distrustfulness, loss of inside and bladder control, unpredictable rest example and expansion in rest, trouble in doing every day essential exercises like perusing, composing, and working with numbers. As AD turns out to be more terrible, indications like expanded nervousness, sadness, fancies are seen.

\subsection{Diagnosis ${ }^{[8,9]}$}

To analyze Alzheimer's dementia, surveying drug history, family ancestry and manifestations is significant. Some Laboratory tests are likewise performed for finding of Alzheimer's dementia known as Brain imaging test which helps in Distinguish between various kinds of degenerative cerebrum sickness and Rule out different causes, like hemorrhages, mind tumors or strokes. The mind imaging advancements frequently utilized are Positron Emission Tomography (PET), Magnetic resonance imaging (MRI) and Computerized tomography (CT).

\subsection{Pathophysiology of $\mathrm{AD}^{[10,11]}$}

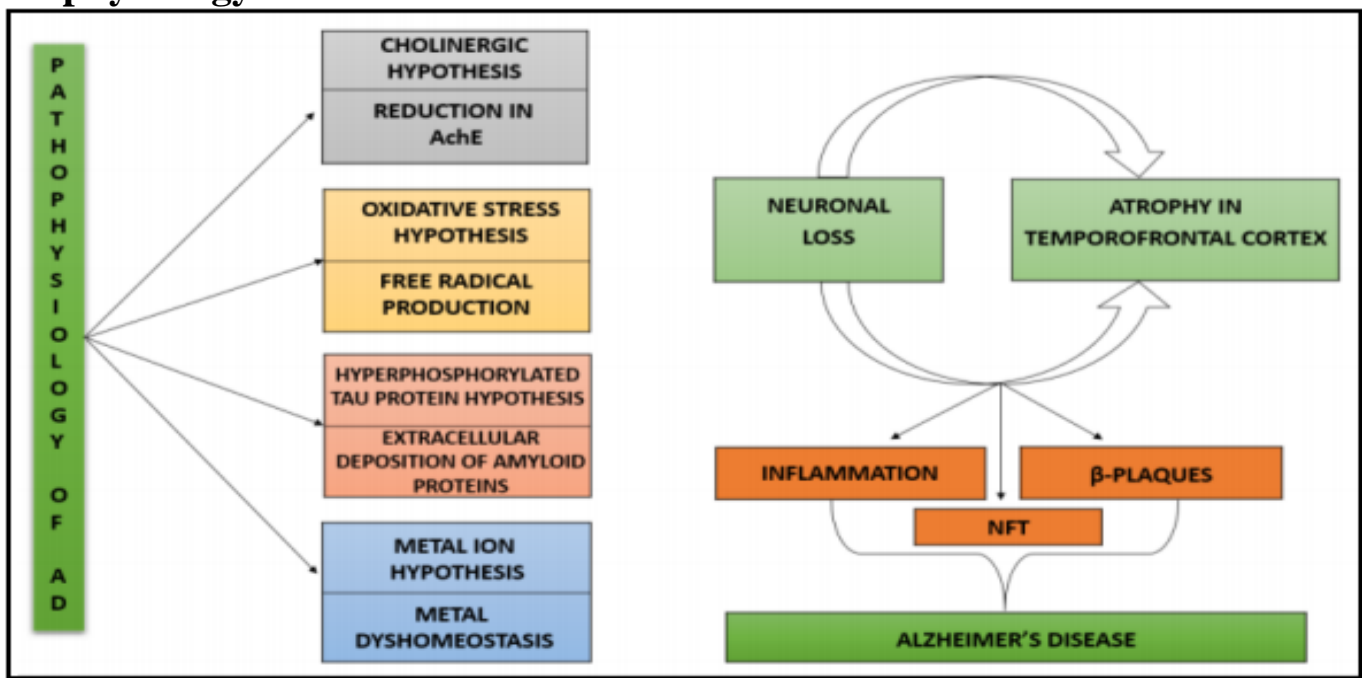

Fig 1: Hypothesis for Pathophysiology of Alzheimer's disease ${ }^{[1]}$

Neuronal misfortune in $A D$ is especially found in amygdala, hippocampus, entorhinal cortex and cortical affiliation spaces of front facing, transient and parietal cortices. It can likewise be seen in subcortical cores like cholinergic basal cores. Mind imaging trial of the patient in $\mathrm{AD}$ uncovers the decay of cerebrum like developed ventricles. The arrangement of feeble plaques and neurofibrillary tangles 
(NFTs) shows the presence of AD. Plaques are shaped because of extracellular statement of filamentous $\beta$-amyloid which is a cleavage result of amyloid forerunner protein. To clarify AD different causative components have been advanced as far as speculation like cholinergic theory, tau speculation, oxidative pressure speculation and metal particle theory. Pathophysiology of $\mathrm{AD}$ is summed up in Fig.1.

\subsubsection{Cholinergic Hypothesis ${ }^{[2,13]}$}

Acetylcholine (ACh) is the main synapse deformity which was found in AD. Apo-lipo-protein E (APOE) is the main genotype related with AD. Choline acetyltransferase and acetylcholinesterase is a significant marker for cholinergic neurons which assumes significant part in union and corruption of ACh. Level of ACh is diminished in space of cerebrum, cortex and hippocampus which are associated with insight and memory. Chiefly cholinergic neurons of core basalis and the entorhinal cortex get influenced. This acknowledgment of acetylcholine job in memory and learning prompts cholinergic theory and it restoratively increments cholinergic movement. The best methodology utilized in $\mathrm{AD}$ is cholinesterase restraint. Tacrine was the principal drug which was endorsed for clinical use in AD. Donepezil (Aricept ${ }^{\circledR}$ ), rivastigmine (Exelon $\AA$ ), and galantamine (Reminyl®) are the three new cholinesterase inhibitors which are as of now accessible.

\subsubsection{Oxidative Stress Hypothesis ${ }^{[14,15]}$}

In age of $\mathrm{AD}$, oxidative pressure assumes significant part in harming CNS. There are a few components which makes oxidative harm CNS this incorporate high substance of unsaturated lipid, high oxygen utilization. Harm by responsive oxygen species (ROS) and receptive nitrogen species held within proper limits by cell reinforcement protection course which comprises of enzymatic and non-enzymatic mixtures. There are different sources which assumes essential part underway of ROS, for example, endogenous sources like mitochondria, cytochrome $\mathrm{P} 450$ and so forth, cancer prevention agent guards like nutrients (A, C and E), glutathione peroxidase, catalase. Because of awkwardness among ROS and cell cancer prevention agent protections there is disappointment of natural capacities. Redox-dynamic $(\mathrm{Fe}, \mathrm{Cu})$ and redoxinactive (Zn) metals are the significant sources because of which free revolutionaries are framed and causes oxidative pressure in AD. Neuron contains huge number of polyunsaturated unsaturated fat and when it responds with ROS brings about atomic apoptosis and lipid peroxidation response, likewise less glutathione in neuron causes oxidative pressure injury.

\subsubsection{Amyloid Cascade and Tau Protein Hypothesis ${ }^{[15,16]}$}

Amyloid theory expresses that there is arrangement of senile plaques (SP) which is framed because of amyloid beta (A $\beta$ ) affidavit created by proteolytic cleavage of amyloid precursor protein (APP) by the activity of $\alpha$-secretase, $\beta$ secretase and $\gamma$ secretase. Two stages are associated with age of A $\beta$ from APP which incorporate $\beta$ secretase and $\gamma$-secretase. Initial step includes age of film bound dissolvable Cterminal part by $\beta$-site APP cleaving enzyme (BACE1). There is further age of $A \beta 40$ and $A \beta 42$ by $\gamma$-secretase, $A \beta 42$ is more neurotoxic. This pathway by which APP is divided is called amyloidogenic pathway. The neurofibrillary tangles (NFTs) is another significant element in AD framed by hyperphosphorylated Tau protein. Tau protein is a profoundly solvent microtubule-associated protein (MAP), which for the most part exists in axon. The primary capacity of tau protein is to keep up with solidness of neurons. Typically the harmony between the phosphorylation and dephosphorylation of Tau protein is kept up with yet when there is arrangement of twofold helix fiber because of total of hyperphosphorylation tau protein, it loses the capacity of associating and balancing 
out microtubules, which prompts passing of neurons.

\subsubsection{Metal particle Hypothesis ${ }^{[17,18]}$}

Metal particles assume a significant part in the upkeep of homeostasis. The connection between metal particles and neurodegenerative sicknesses has drawn in much consideration as of late. The cerebrum is wealthy in metals that go about as fundamental cofactors in metalloproteins to takes part during the time spent digestion, the convergence of metal particles in the mind is firmly directed through the blood mind obstruction, when the blood cerebrum boundary of metal particle guideline framework corruption, metal particle transport brokenness, metal particles (iron, copper, manganese, aluminum, zinc, and so on) start to influence the oxidative pressure reaction of mitochondria and some unacceptable collapsing proteins, and at last lead to neurodegeneration. Studies have demonstrated that aluminum, zinc, copper and iron can prompt changes in the compliance of the $A \beta$ protein. Aluminum can prompt the gathering of $A \beta$ and Tau protein, aluminum and copper are associated with the interaction of the improvement of nerve irritation. The expanded degrees of iron, aluminum and copper in the matured human mind might mirror the connection among age and neurodegenerative illnesses Current Evidence shows changes in the harmony of redox progress metal; predominantly copper (cu), iron $(\mathrm{Fe})$, and other follow metals. Their levels in the mind are observed to be high in AD. In other neurodegenerative issues, $\mathrm{Cu}$, Magnese, aluminum, and Zinc are included.

\section{Treatment of $\mathrm{AD}^{[19-25]}$}

There are two kinds of medicines accessible to oversee AD; ordinary pharmacotherapy and other is utilization of restorative spices for the treatment of AD.

Ordinary pharmacotherapy for AD which is endorsed by the Food and drug administration (FDA) are mostly of two sorts:
- Acetylcholinesterase inhibitorsGalantamine, , Donepezil, Rivastigmine, Huperzine A, Tacrine

- N-methyl-D-aspartate glutamate antagonist (NMDA)- Memantine

\subsection{Acetylcholinesterase inhibitors ${ }^{[19,20,21]}$}

The main medications which were endorsed by FDA in treatment of Promotion is acetylcholinesterase inhibitors. It acts by confining the breakdown of acetylcholine by cholinesterase chemical and accordingly there is expansion in centralization of acetylcholine at destinations of neurotransmission. In 1993, FDA supported the primary medication Tacrine as acetylcholinesterase inhibitors in treatment of Advertisement. Be that as it may, presently Tacrine is once in a while utilized because of its hepatotoxicity. USFDA likewise endorsed three additional medications for treatment of patients experiencing gentle to direct $\mathrm{AD}$ for example donepezil, rivastigmine, and galantamine. These three medications doesn't shows hepatotoxicity as tacrine. There are two medications in cholinesterase inhibitors which are gotten from regular item for example Galantamine and Huperzine A.

Galantamine is a tertiary alkaloid which was separated from Galanthus species (snow drop) and furthermore in different plants having a place with Amaryllidaceae plant family like Narcissus species (Narcissus spp). It is a concentrate of blossoms and bulbs of daffodil and lilies. Galantamine is a reversible Ach inhibitor which can likewise be arranged artificially by different techniques. It forestalls the cytotoxicity brought about by total of $A \beta$ and in this way works on psychological brokenness and manages the cost of neuronal security. By allosteric regulation of pre-and post-synaptic nicotinic receptors it upgrades focal neurotransmission. After oral organization galantamine get quickly ingested. It is utilized to treat gentle to direct AD. Galantamine was first detailed as prompt delivery arrangement, since of half- 
existence of medication is 7hours portion ought to be allowed double a day

Rivastigmine is a carbamate derivative, it restrains both $\mathrm{AChE}$ and BuChE. It is supposed to be pseudoirreversibe inhibitor since it structures carbamoylated complex with the dynamic site serine. Rivastigmine was observed to be the solitary medication where cytochromeP450 isoenzymes isn't include in its digestion and in this way it limits drugdrug cooperation. It is showcased under the business trademark Exelon. The wide range of various medications utilized in Throb are accessible in oral structure while rivastigmine is accessible as transdermal fix and endorsed for gentle to direct Promotion in a few nations like USA, Europe, Latin America and Aisa. During clinical preliminaries it show incidental effect, influencing the gastrointestinal tract. Rivastigmine have half-existence of about 3 hour in fix and about 1 hour in capsule.

Donepezil is the one more acetylcholinesterase inhibitor utilized in treatment of gentle to direct AD in 1997. Donepezil is the piperidine based subordinate. It is non-covalent reversible inhibitor of acetylcholinesterase, it structures bond with protein and at the same time communicates with the PAS. It shows less selectivity towards BChE. The subsequent expansion in acetylcholinen in the CNS for synaptic transmission, it is more particular towards cerebral cortex. Tacrine is generally ingested after oral organization, half-life is upto 70hours likewise it is utilized in every one of the phases of $\mathrm{AD}$. It shows not many incidental effects like queasiness, loose bowels, anorexia.

Huperzine An is a quinolizindine alkaloid which is separated from a plant Huperzia serratum having a place with family Lycopodiaceae. It is reversible and compelling inhibitor of $\mathrm{AChE}$ which crosses blood brain barrier (BBB) and give security against neuronal harm. It builds level of acetylcholine in cerebrum by restraining acetylcholinesterase movement and in this way work on intellectual capacity in understanding with AD. It shows longer term of activity when contrasted and galantamine, tacrine and donepezil. Huperzine An enjoy different benefits like higher oral bioavailability, less unfavorable response and so on.

Tacrine was the primary medication which was endorsed in the treatment of AD by FDA in 1993 as acetylcholinesterase inhibitor. Tacrine (tetrahydroaminoacridine, THA) advertised under the name Cognex ${ }^{\circledR}$. It is non-serious (reversible) inhibitor of $\mathrm{AChE}$ and BChE. It shows cholinergic harmfulness because of its greater explicitness towards BChE when contrasted with AChE. Tacrine represses monoamine oxidases by connection with muscarinic receptor. In the amalgamation of bivalent THA more effordsare done to expand its particularity towards PAS site of AChE. The utilization of medication was diminished because of rise of liver transaminases which comprises of alanine aminotransferase (ALT) and aspartate aminotransferase, as a result of these the danger of liver injury was expanded (hepatotoxicity). Hence, tacrine was removed from the market. Tacrine is retained from GIT and its pinnacle plasma fixation increment with expansion in oral portion, likewise whenever taken with food its focus diminishes upto $30 \%$

\subsection{N-methyl-D-aspartate glutamate antagonist (NMDA antagonist) ${ }^{[22,23,24]}$}

NMDA receptor adversary was supported by FDA in October2003, memantine is the lone medication in NMDA which was endorsed by FDA in treatment of AD. Mitochondrial brokenness and calcium over-burden was a consequence of Glutamate interceded excitotoxicity, expanding nitric oxide age which frames undeniable degree of oxidants and neuronal apoptosis. This total interaction is impeded by NMDA receptor antagonist.

Memantine

(1-amino-3,5dimethlyadamantane) is an individual from the aminoadamantane class of natural 
atoms. In 2002, memantine was endorsed as a remedial medication in treatment of moderate to serious $\mathrm{AD}$ by the European Agency for the Evaluation of Medicinal Products (EMEA), trailed by USA Food and Drug Administration (FDA) in 2003.Memantine is the solitary medication which is remembered for NMDA having low fondness, voltage reliant, non-cutthroat bad guy of NMDAR is recommended in AD alongside AChEI. At high focus memantine can restrain systems of synaptic versatility. It secures neurons by diminishing glycogen synthase kinase $3 \beta$ (GSK-3 $\beta$ ) action by constricting tau phosphorylation. Contingent on Mini-Mental State Examination score < 20. While recommending memantine to patient with renal disappointment or epilepsy alert ought to be taken. The halfexistence of medication is 60-100 hours, beginning portion of memantine is $5 \mathrm{mg}$ once every day, with an objective portion of $20 \mathrm{mg} /$ day.

\subsection{Gamma-secretase inhibitors ${ }^{[22]}$}

Interesting proof demonstrates that sub-ongoing treatment (twice-aday for 12 days) and intense organization of semagacestat (LY-450139), a gammasecretase inhibitor, at a portion that diminishes $A \beta$ levels creates a decrease in force of hippocampal $\theta$ action evoked by electrical incitement of NPo in mice.

\subsection{Muscarinic initiation ${ }^{[25,26]}$}

The general impact of the actuation of muscarinic acetylcholine receptors delivers an expansion in $\theta$ and $\gamma$ motions for certain mind boggling explicit elements. In an investigation performed by Lebois et al, intense therapy with BQCA, a M1 receptor positive allosteric modulator at a portion that has been identified with memory execution improvement, increments intra hippocampal (CA3-CA1) intelligibility of $\theta$ and $\gamma$ motions contrasted with control movement yet diminishes spiking explicitness of spot cells comparative with hippocampal $\theta$ motions recorded in uninhibitedly moving rodents in various formed conditions. In a similar report, intense therapy with the M1 receptor agonist VU0364572 produces an increment in $\theta$ and $\gamma$ swaying power contrasted with control action without changing the particularity spiking of spot cells comparative with hippocampal $\theta$ motions. Extra proof additionally demonstrates that the intense use of another M1 specific agonist (77-LH28-1) creates the acceptance and increment of $\gamma$ motions in hippocampal cuts.

\subsection{Nicotinic enactment ${ }^{[24]}$}

As a general impact, pharmacological initiation of nicotinic acetylcholine receptors (nAChRs) actuates an expansion in both $\theta$ and $\gamma$ motions. The intense organization of nicotine to hippocampal cuts acquired from about a month old rodents builds the synchronization and autocorrelation of $\gamma$ motions created by $100 \mathrm{~Hz}$ incitement of Schaffer's insurances. Curiously, when applied intensely at low fixation to hippocampal cuts got from about a month old Wistar rodents, nicotine builds the force of $\gamma$ motions instigated by kainate however has the contrary impact when the focus is expanded. At the point when the impact of explicit initiation of $\mathrm{nAChRs}$ was tended to, an overall expansion in force of both $\theta$ and $\gamma$ motions. Intense organization of the particular $\alpha 7-n A C h r$ agonist PNU-282987 to anesthetized rodents expands the force of $\theta$ hippocampal motions delivered by incitement of the nPO and advances an expansion in the $\theta-\gamma$ coupling of hippocampal action. Besides, PNU-282987 organization additionally advances an increment in the force of kainate-initiated $\gamma$ motions in rodent hippocampal cuts. Moreover, intense organization of the specific $\alpha 7-n A C h r$ agonist FRM-17874 to anesthetized rodents builds the force of $\theta$ hippocampal motions created by nPO incitement and improves the balance file among $\theta$ and low- $\gamma$ action; likewise with the impact on power portrayed in anesthetized mice. At long last, intense organization of the $\alpha 4 \beta 2-n A C h r$ agonist RJR2403 advances an expansion in the force of $\gamma$ motions 
delivered by kainate in rodent hippocampal cuts.

\subsection{Antiepileptic drugs ${ }^{[25]}$}

Concerning antiepileptic drugs, intraperitoneal infusion of a high portion of carbamazepine produces a decrease in force of unconstrained $\gamma$ oscillatory action recorded by EEG in vivo in APP-PS1 transgenic mice. A similar report found that phenytoin treatment improves $\theta$ wavering force and surprisingly a decrease in the portion is as yet equipped for expanding $\gamma$ swaying power. Moreover, valproic corrosive treatment delivers a dumbfounding outcome of $\theta$ oscillatory action recorded by EEG in APP-PS1 transgenic mice: while the low portion increments $\theta$ oscillatory action, a higher portion decline it, recommending that definite adjusting of the portion utilized is fundamental for produce the gainful results of antiepileptics for oscillatory action in AD models.

Different treatments Among different systems utilized as potential infection changing specialists we tracked down an extraordinary assortment of restorative medications and targets. For instance, the effective utilization of GABA to the cortex of 3-4 old APP/PS1 mice, which overexpress APP and have the Delta E9 transformation on PS1, reestablishes the force of slow-wave oscillatory movement to levels like WT mice. Likewise, initiation of G-protein-gated internally correcting potassium channels (Kir3/Girk) with ML297 forestalls the decrease in force of $\theta$ and $\gamma$ hippocampal action recorded in vivo in mice intra cerebroventricularly infused with $A \beta$ thus forestalling memory changes in a clever item acknowledgment test. Such preventive impacts of ML297 are underlain by reestablishing the sensitivity shortfalls delivered by $\mathrm{A} \beta$ on hippocampal network.In different examinations, i.p. infusions of SA5-CH, which is a double GLP-1/GIP receptor agonist, reestablished the force of hippocampal $\theta$ oscillatory movement in rodents i.c.v. infused with streptozotocin. At last, constant treatment with melatonin has all the earmarks of being ready to pevent the decrease of $\theta$ swaying power and reestablishes the memory in $\mathrm{A} \beta$ intrahippocampal infused WT rodents.

\section{Neuroprotective Natural Products from Medicinal Plants for $\mathrm{AD}^{[28-37]}$}

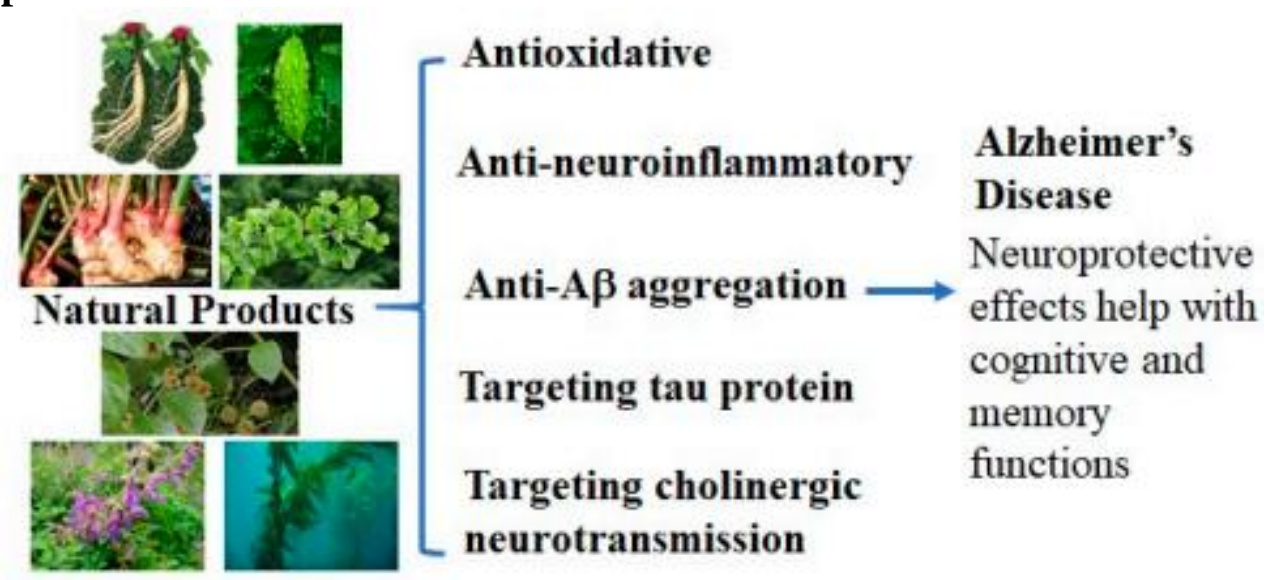

Fig.2: Neuroprotective Effects from Natural Products for AD ${ }^{[2]}$

Medicinal plants have been found to have the option to diminish $\mathrm{AD}$ progress and manifestations. Both the concentrates and the dynamic individual mixtures from restorative plants have been seriously researched for their impacts on AD. The dynamic mixtures detached from therapeutic plants, for example, phenolic lignans, flavonoids, tannins, and polyphenols, just as triterpenes, sterols, and alkaloids, have shown different valuable neuroprotective capacities, including cell reinforcement, against neuroinflammatory, hostile to amyloidogenic, hostile to tau collection, and 
anticholinesterase exercises. A portion of these dynamic mixtures, either as single parts like curcumin, melatonin, resveratrol, and nutrients $\mathrm{C}$ and $\mathrm{E}$, or as natural concentrates, for example, matured garlic remove, Ginkgo biloba concentrate, and green tea have been assessed in AD patients with positive outcomes.Such spices are Ashwagandha (Withania somnifera), Turmeric (Curcuma longa), Shankhpushpi (Convolvulus pluricaulis), Ginkgo Biloba, Saffron (Crocus sativus), Brahmi (Bacopa monnieri), Gotu Kola (Centella asiatica), Triphala (emblica officinalis), Cat's hook (uncaria tomentosa).

\subsection{Ashwagandha (Withania somnifera) [31,32]}

Ashwagandha which otherwise called Indian ginseng or winter cherry, is quite possibly the most conspicuous spices endorsed as a cerebrum rejuvenator for AD. Withania somnifera is a little woody bush having a place with the family Solanaceae and is broadly filled in India. Its blossoms are greenish or yellowish in shading and around one centimeter long. The major phytoconstituents of W. somnifera are isopellertierine, anferine, withanolides, withaferins, sitoindoside VII and VIII and withanoloides. Other substance compounds are withanine, somnine, somniferine, somniferinine, withananine, choline, pseudo-withanine, tropine, pseudo-tropine, 3-a-gloyloxytropane, and cuscohygrine. Ashwagandha have cell reinforcement action, free revolutionary searching movement, just as an insusceptible framework promoter. The plant concentrate of Ashwagandha is utilized in therapy and counteraction of different illnesses like Arthritis, feebleness, amnesia, disease and neurodegenerative issues. The instrument of activity of Ashwagandha in people isn't clear however It works on psychological conduct in rodents exposed to oxidative harm that happens in $\mathrm{AD}$ and can turn around gathering of $\beta$ Amyloid peptides (A $\beta)$ ensnared in the illness. Creature studies have shown that the Sitoindosides
VII-X and Withaferina A (glycowithanolides) are the dynamic phytophenols, answerable for the instrument of expanded cortical Muscarnic acetylcholine limit, with a balance of cholinergic neurotransmission. Atomic displaying studies showed that Withanamides-A, C Uniquely tie to dynamic moiety of beta-amyloid and forestall fibril arrangement. Fluid concentrate expands the cholinergic movement, while Methanol separate causes neuritis outgrowth in portion and time subordinate way in human neuroblastoma cells. A forthcoming, fake treatment controlled examination announced that treatment with ashwagandha-root separate further developed prompt and general memory works and upgraded chief capacity, consideration and data handling speed in grown-ups with a gentle psychological debilitation. There is restricted information accessible on the clinical utilization of Withania for psychological impedance. By the investigations directed till now, it was seen that the arrangements and concentrates of W. somnifera root didn't cause any harmfulness even on ongoing treatment.

\subsection{Brahmi (Bacopa monnieri) ${ }^{[33]}$}

Bacopa monnieri (B. monniera), is a little, enduring crawling spice with various branches, little elongated leaves and light purple or white blossoms having a place with the Scrophulariaceae. It is Traditionally utilized for tension alleviation, as a tonic for the mind to improve learning and memory advancement, and anticipation of epilepsy. The principle synthetic mixtures of $\mathrm{B}$. monniera are triterpenoid saponins known as bacosides. The bioactive phytochemicals present in this plant incorporate saponins, bacopasides III, IV, V, bacosides An and B, bacosaponins $\mathrm{A}, \mathrm{B}, \mathrm{C}, \mathrm{D}, \mathrm{E}$, and $\mathrm{F}$, alkaloids, sterols, betulic corrosive, polyphenols, and sulfhydryl compounds, which might be liable for the neuroprotective jobs of the plant. The bacosides additionally improves kinase movement, neuronal union, and reestablish 
synaptic action. Bacopa monnieri has different organic exercises like anticonvulsant, stimulant, anxiolytic, pain relieving, mitigating, cell reinforcement, antimicrobial, antiulcerogenic, hostile to Helicobacter pylori, adaptogenic, antineoplastic, bronchodilatory, hepatoprotective and immunostimulatory. During the investigations, Bhrami separates shows security of neurons from betaAmyloid incited cell demise by smothering cell acetyl cholinesterase movement. It has additionally switched activities like consumption of acetyl choline, decrease in Choline acetyl Transferases, decline in Muscarnic cholinergic receptor restricting in cerebrum and hippocampus. During the investigations, Individuls over 55 years old with memory hindrance, normalized Bacopa extricate $125 \mathrm{mg}$ was given twice day by day for 12 weeks in a twofold visually impaired, fake treatment controlled way. There was a critical improvement in mental control, coherent memory and matched related learning.

\subsection{Cat's claw (Uncaria tomentosa) ${ }^{[34]}$}

Cat's hook is a famous home grown enhancement got from a tropical vine,belongs to the family Rubiaceae. It fills quickly in the Amazon rainforest and in other tropical spaces of South and Central America. Two predominant types of feline's paw are Uncaria tomentosa and Uncaria guianensis, both are for their mitigating properties in South America. Plant of leaves contain high measure of oxindole alkaloid content when contrasted with stem, bark and branches. U. toemntosa contains recently distinguished polyphenolic compound specifically proanthocyanidins that have both plaque and tangle with lessening and inhibitory impact. Proanthocyanidin B2 reduce the mind plaqueand improves transient memory; it is more grounded inhibitor of cerebrum aggravation. Subsequent to treating 8-month-old mice with the $\mathrm{CC}$ concentrate for 14 days, decrease in the $A \beta$ load (by 59\%) and plaque number (by 78\%) in the hippocampus and cortex was noticed, it additionally shows decrease in astrocytosis and microgliosis and further develop hippocampus subordinate memory. A few parts of concentrate likewise crosses the $\mathrm{BBB}$ and enter mind parenchyma. It is considered as expected plant in treatment of $\mathrm{AD}$ as it is solid eliminator of $\mathrm{A} \beta$ plaques.

\subsection{Ginkgo Biloba ${ }^{[35]}$}

It is regularly called as ginkgo, it has a few uses in conventional medications and as a wellspring of food. This plant is local to china and south Japan. Concentrates of ginkgo leaves contain various sorts of phytochemicals incorporate phenolic corrosive, proanthocyanidins, flavonoid glycosides (Myricetin, kaempferol, isohamnetin, quercerin), Terpens, ginkolides, biobalides, ginkgo biflavones and alkylphenols. Ginkgo biloba (Gb) has been at the center of attention fundamentally for its expected job in treating $\mathrm{AD}$. The flavonoids and Terpens in the concentrate essentially hinder acetylcholinesterase movement in cerebrum. Ginkolides is a strong enemy of oxidant with cholinergic and neuroprotective exercises henceforth it ensures against $A \beta$ Protein incited oxidative harm. $\mathrm{Gb}$ is utilized as an underlying treatment for beginning phase $\mathrm{AD}$ and vascular dementia. Treatment with $\mathrm{Gb}$ remove upgraded memory maintenance in youthful and old rodents likewise momentary memory in mice is improved. A few examinations demonstrate that ginkgo postpones the movement of $\mathrm{AD}$ and is just about as viable as the cholinesterase inhibitors for treating $\mathrm{AD}$. $\mathrm{Gb}$ remove likewise further develops ADLs among AD people and is liked over other AD prescriptions in view of its insignificant antagonistic impacts.

\subsection{Shankhpushpi (Convolvulus pluricaulis) ${ }^{[36]}$}

Shankhpushpi is a typical plant found in Northern India, it has different species, for example, Convolvulus pluricaulis (CP), Convolvulus microphyllus, Evolvulus alsinoides, and Clitoria ternatea 
(CT), it has a place with family Convulvulaceae. The entire plant of shankhpushpi is utilized as nervine tonic to further develop memory and intellectual capacity. Leaves are circular fit, the branches are $30 \mathrm{~cm}$ long and plant comprises of blue blossom. Plant contains different phytochemicals like alkaloids (shankhpushpine and convolamne), glycosides, flavonoids, coumarines additionally there are some different constituents which are available in plant are Sitosterol, Hydroxy cinnamic corrosive, Octacosanol. Hippocampus is a fundamental locale of cerebrum which is answerable for learning and memory work shows portion subordinate expansion in acetyl cholinesterase action. Cholinergic and glutamatergic flagging is expanded by gathering of neutraceuticals known as racetams. An ethanolic concentrate of Convolvulus pluricaulis when tried in-vitro shows cell reinforcement action. It is utilized in the treatment of dementia, OCD, fears, Insomnia. It quiets the nerves by controlling pressure chemicals, Adrenaline and cortisol. Ethanolic extricate, ethyl concentrate of it and watery portion fundamentally further developed learning and memory in rodents.

\subsection{Turmeric (Curcuma longa) ${ }^{[37]}$}

It is a lasting herbaceous plant normally known as turmeric having a place with the family Zingiberaceae. It is utilized in Asia for millennia in Ayurveda, Siddha, Unani, conventional Chinese medication. India is the world's biggest maker, customer and exporter of turmeric. Turmeric is gotten from the rhizome of the plant, and is generally utilized in India as a food enhancing and shading specialist. The turmeric powder contains $60-70 \%$ starches, $6-13 \%$ water, $6-8 \%$ proteins, $3-7 \%$ fundamental oils, $2-7 \%$ dietary fiber, $1-6 \%$ curcuminoids. The radiant yellow shading that rhizome is for the most part due to the polyphenolic compounds called curcuminoids. The dynamic constituents of turmeric are turmerone oil, curcumin, demethoxycurcumin

(DMC), bisdemethoxycurcumin (BDMC), and cyclocurcumin Turmeric is mitigating, sterile, and antibacterial and has for quite some time been utilized to treat a wide assortment of conditions including liver detoxification, to forestall contamination and aggravation, to adjust cholesterol levels, to treat sensitivities, to animate assimilation, and to help invulnerability. It has been demonstrated that curcumin utilization is helpful in the treatment of AD and dementia it likewise can diminish the arrangement of Amyloid plaques and postpones corruption of neurons Curcuminoids are demonstrated to have solid cancer prevention agent activity exhibited by the restraint of the development and engendering of free revolutionaries.

Curcumin is the head curcuminoid whose mitigating property is related with diminished danger of AD. A few investigations led on creatures having $\mathrm{AD}$, have announced improvement in intellectual capacity in the curcumin-treated gathering. Scientists property the improvement to curcumin's capacity to bring down $\mathrm{A} \beta$ plaque levels just as to its mitigating and cell reinforcement properties. Curcumin likewise turns around psychological disabilities in different creature models of AD. Higher portions of curcumin are more successful contrasted with the lower dosages. When curcumin was given in blend with piperine, enhancements in perception were more prominent particularly against persistent illnesses. Curcumin structures solid buildings with metals like copper, zinc, or iron and squares metal-set off $\mathrm{A} \beta$ conglomeration, harmfulness, and irritation as these metals are moved in the AD mind and trigger amyloid collection or oxidative neurotoxicity.

\subsection{Other Medicinal Plants for $\mathbf{A D}^{[38]}$}

There are a few other restorative plants that play a part in the anticipation or treatment of $\mathrm{AD}$ yet a few investigations shows that their job in AD is exceptionally restricted. These plants incorporate vacha 
(Acorus calamus), guduchi (Tinospora cordifolia), guggul (Commiphora wightii), jatamansi (Nardostachys jatamansi), jyotismati (Celastrus paniculatus), rosemary (Rosmarinus officinalis), Green tea (Camellia sinensis), St john's wort (Hypericum perforatum), sage (Salvia spp), Rhodiola rosea, Moringa oleifera, shilajit, and lemon medicine. Further investigations are as yet continuing for the viability of these medications.

\subsection{Nuts Including Almond, Hazelnut, and Walnut ${ }^{[39,40]}$}

All of these nuts, considered as useful for the mind, apply neuroprotective impacts that add to sharpness, focus, and memory. Many examinations have demonstrated that nuts contain a rich grid of bioactive synthetic substances and could have the ability to help neuronal capacity in the cerebrum. The connection between nut utilization, worked on intellectual execution, and brought down frequency of $\mathrm{AD}$ has been affirmed by a few examinations, supporting their AD preventive properties.

Adding hazelnut portion into rodents' eating routine prompted improved memory, diminished tension, and enhanced neuroinflammation and apoptosis. As a dietary enhancement, hazelnut has been affirmed to help sound maturing. Almond supplementation in rodents' eating regimen for one to about fourteen days altogether switched amnesia actuated by scopolamine. This impact was accepted to have come about because of diminished AChE movement just as brought down cholesterol and fatty substance levels, along with somewhat expanded glucose level. Adding almond glue for about a month in rodents' eating regimen altogether further developed learning and memory with upgraded mind tryptophan monoamine levels and serotonergic turnover in the cerebrum. Fundamentally further developed memory maintenance was likewise seen in one more investigation with about a month of almond organization to rodents, which was ascribed to the raised degree of Ach.
Almond and pecan supplementation for about a month lessened cadmiuminitiated memory debilitation in rodents, conceivably through cholinergic and cancer prevention agent exercises. A pecan advanced eating routine has been displayed to further develop psychological and engine execution. Its neuroprotective impacts are accepted to be related with the amazingly constricted articulation of proinflammatory cytokines, diminished degree of $\mathrm{AChE}$, essentially reestablished levels of cancer prevention agent catalysts, and decreased articulation of NF- $\kappa B$. Pecan could likewise expand the quantity of Ach receptors and upregulate articulation of ChAT. In rodent PC12 cells, the peptides from defatted pecan protein displayed critical free revolutionary searching and cytoprotective exercises against oxidative harm. Pecan extricate hindered $A \beta$ fibril arrangement and diminished $A \beta$-interceded cell passing. All noticed neuroprotective impacts related with nuts are preventive for AD.

\section{Neuroprotective Natural Products from Marine Sources for $\mathrm{AD}^{[41,42]}$}

Marine normal items, created under unfriendly conditions and containing surprising designs, have been a rich hotspot for the treatment of various sicknesses including $\mathrm{AD}$. Various marine normal items, along with their determined engineered analogs, showed great viability against $\mathrm{AD}$. In this manner, marine normal items are effectively pursued as neuroprotective specialists for AD.

Marine Macroalgae (Seaweeds) Marine macroalgae which are plant-like organic entities ordinarily found in waterfront regions contain a wide assortment of bioactive synthetic substances, for example, polyphenols, polysaccharides, shades, amino acids, peptides, and proteins. The medical advantages related with marine macroalgae and their bioactive mixtures have been summed up in a few great audit papers. The methanolic concentrate of Eisenia bicyclis (Kjellman) Setchell, an enduring earthy 
colored ocean growth, showed neuroprotective action along with its ethyl acetic acid derivation and n-butanol subfractions by diminishing intracellular ROS creation in PC12 cells prompted with A $\beta 25-35$. The parts that added to this action were accepted to be phlorotannins eckol, phlorofucofuroeckol A, and 7-phloroeckol. One more earthy colored kelp, Ishige foliacea has been found to contain a phlorotannin-rich division that further developed memory disability in mice through the added substance or synergistic impact from a few instruments including lessening cerebrum AChE movement, smothering oxidative pressure, and enacting the ERK-BDNF-CREB flagging pathway. This load of studies showed that marine macroalgae present extraordinary choices for $\mathrm{AD}$ avoidance and treatment through securing neuron harm and further developing memory disability by various pathways to display neuroprotective action which might result from the impacts of its bioactive parts and the added substance or synergistic impacts of those bioactive parts.

\subsection{Spirulina Cyanobacteria ${ }^{[41]}$}

Mostly alluded to as blue green growth, Cyanobacteria are really prokaryotic living beings all the more firmly identified with microorganisms with fascinating pharmacological properties. Spirulina platensis is a cyanobacterium known to have plentiful nutritive components like carotenoids, polysaccharides, polyunsaturated unsaturated fats, nutrients, minerals, and protein. It has been accounted for that $\mathrm{S}$. platensis protein remove was a powerful cancer prevention agent which had the option to search free extremists with its chelating limit and forestall revolutionary intervened cell demise in vitro. A subsequent report uncovered that the fluid concentrate of S. platensis and its dynamic part C-phycocyanin could lessen cytotoxicity and hinder the statement of aggravation related qualities like $\mathrm{COX}-2$,
TNF- $\alpha$, IL- 6 , and iNOS in vitro, proposing its AD preventive impacts.

Another cyanobacterium, Spirulina maxima, contains numerous physiologically dynamic synthetics including carotenoids, polysaccharides, chlorophylls, Cphycocyanin, and nutrients. Its concentrate was found to enhance learning and memory weaknesses in an AD mice model, ensnaring its potential in AD treatment. This neuroprotective impact was accepted to come from diminished articulation levels of hippocampal A $\beta 1-42$, APP, and BACE1, just as diminished AChE movement, stifled hippocampal oxidative pressure, expanded BDNF level, and enacted BDNF/PI3K/Akt flagging pathways. In one more examination with PC12 cells treated with A $\beta 1-42$, the Spirulina maxima extricate forestalled $A \beta$ actuated oxidative pressure and cell demise through the initiation of BDNF flagging.

\subsection{Thalassospira Profundimaris ${ }^{[42]}$}

A screening program with 225 marine bacterial concentrates taking a gander at both their poisonousness and neuroprotective properties recognized a few marine bacterial concentrates as promising leads, with Thalassospira profundimaris being the most intense one. Its rough concentrate had the option to save synaptic design in vitro. An in vivo follow-up examination uncovered its $\mathrm{AD}$ preventive limit since it could obstruct the cell cyclerelated neuron demise. While none of the parts got from the unrefined concentrate shown neuroprotective movement as strong as the entire concentrate, it was estimated that the synergistic activity of a few parts may be answerable for the general impacts.

\section{CONCLUSION}

The etiology of the neurodegenerative illnesses isn't actually known, the pharmaceutical industry is confronting different difficulties in the medication revelation measure. Including ayurvedic system there are modest number of pharmacological specialists accessible to deal with the manifestations of AD. Due to 
lesser side effects ayurvedic drugs are becoming familiar in present days. Neuroprotective methodologies including numerous mechanism of action are significant for the anticipation and treatment of $\mathrm{AD}$. In $\mathrm{AD}$ drug disclosure Natural product blends or concentrates, with numerous bioactive mixtures and the capacity to apply various neuroprotective mechanisms are essential. With more viable and extensive quality control rules created to guarantee the wellbeing and viability of regular item treatments, just as new methodologies and techniques to assist with advancing the CNS access of these neuroprotective specialists, like the fuse of nanotechnology in the conveyance of normal items, normal item treatment could assume a fundamental part in the prevention and treatment of $\mathrm{AD}$.

\section{Acknowledgement: None}

\section{Conflict of Interest: None}

\section{Source of Funding: None}

\section{REFERENCES}

1. Yash R Menghani, Dhanshree M Bhattad, Kamala K Chandak, Jayshree B Taksande and Millind $\mathrm{J}$ Umekar, A Review: Pharmacological and herbal remedies in The Management of Neurodegenerative disorder (Alzheimer's); International Journal of Pharmacognosy and Life Science 2021; 2(1): 18-27. https://doi.org/10.33545/ 27072827.2021.v2.i1a.23

2. Xin Chen 1,Joshua Drew, Wren Berney and Wei Lei; Neuroprotective Natural Products for Alzheimer's Disease; Cells 2021, 10, 1309. https://doi.org/10.3390/cells10061309

3. Arturo G. Isla, Hugo Balleza-Tapia, Andr'e Fisahn, Efficacy of preclinical pharmacological interventions against alterations of neuronal network oscillations in Alzheimer's disease: A systematic review; Experimental Neurology 343 (2021) 113743 https://doi.org/10.1016/j.expneurol. 2021.113743

4. Hippius, H.; Neundorfer, G. The discovery of Alzheimer's disease. Dialogues Clin. Neurosci. 2003, 5, 101-108.
5. James, B.D.; Leurgans, S.E.; Hebert, L.E.; Scherr, P.A.; Yaffe, K.; Bennett, D.A. Contribution of Alzheimer disease to mortality in the United States. Neurology 2014, 82, 1045-1050.

6. Silva, M.V.F.; Loures, C.M.G.; Alves, L.C.V.; de Souza, L.C.; Borges, K.B.G.; Carvalho, M.D.G. Alzheimer's disease: Risk factors and potentially protective measures. J. Biomed. Sci. 2019, 26, 33.

7. Cummings, J., Lee, G., Ritter, A., Sabbagh, M., Zhong, K., 2019. Alzheimer's disease drug development pipeline: Alzheimer's Dement. Transl. Res. Clin. Interv; 2019, 5, 272-293. https://doi.org/10.1016/j.trci.2019.05.008.

8. McDermott, B., Porter, E., Hughes, D., McGinley, B., Lang, M., O'Halloran, M., Jones, M., Gamma band neural stimulation in humans and the promise of a new modality to prevent and treat Alzheimer's disease. J. Alzheimers Dis; 2018, 65, 363392. https:// doi.org/10.3233/JAD-180391.

9. Patwardhan B. Ayurveda: the 'designer' medicine: a review of ethno pharmacology and bioprospecting research. Indian Drugs 2000; 37: 213-27.

10. Parul Agarwal, Shashi Alok, Amreen Fatima and Prem Prakash Singh, Herbal Remedies For Neurodegenerative Disorder (Alzheimer's Disease): A Review, IJPSR 2013; 4(9):3328-3340.

11. Robert Briggs, Sean $P$ Kennelly and Desmond O'Neill, Drug treatments in Alzheimer's disease, Clinical Medicine 2016;16(3):247-53.

12. Agarwal P, Alok S, Fatima A and Singh PP. Herbal remedies for neurodegenerative disorder (Alzheimer's disease):International Journal of Pharmaceutical Sciences and Research 2013;4 (9):3328.

13. Mayeux R. Early Alzheimer's disease. N Engl J Med 2010;362(4):2194-2201.

14. V. Rajmohan, and B. Raghunath1, Neurobiology of Alzheimer's disease, Indian J Psychiatry. 2009; 51(1):55-61.

15. Liu Z, Zhang A, Sun $H$ et al. Two decades of new drug discovery and development for Alzheimer's disease. RSC Advances 2017;7(10):6046-6058.

16. Prakash A, Dhaliwal GK, Kumar $P$ et al. Brain biometals and Alzheimer's diseaseboon or bane? Int J Neurosci 2017;127 (2):99-108. 
17. Xiaoting Sun, Lan Jin, Peixue Ling, Review of drugs for Alzheimer's disease, Drug Discoveries \& Therapeutics 2012;6(6):285290.

18. Ago Y, Koda K, Takuma K, Matsuda T. Pharmacological aspects of the acetylcholinesterase inhibitor galantamine. J Pharmacol Sci. 2011; 116:6-17.

19. Kurz A, Farlow $M$ \& Lefèvre G. Pharmacokinetics of a novel transdermal rivastigmine patch for the treatment of Alzheimer's disease: a review. International Journal of Clinical Practice 2009;63(5):799805.

20. Aranda-Abreu GE, Hernandez-Aquilar ME, Denes JM, Garcia Hernandez LI, Rivero MH. Rehabilitating a brain with Alzheumer's: A proposal. Clin Interv Aging. 2011; 6:53-59.

21. Robert $\mathbf{J}$ van Marum, Update on the use of memantine in Alzheimer's disease, Neuropsychiatr Dis Treat 2009; 5:237-247.

22. Hajos,' M., Morozova, E., Siok, C., Atchison, K., Nolan, C.E., Riddell, D., Kiss, T., Hajos- ' Korcsok, Effects of the $\gamma$ secretase inhibitor semagacestat on hippocampal neuronal network oscillation. Front. Pharmacol. 2013, 72. https://doi.org/ 10.3389/fphar.2013.00072.

23. Lebois, E.P., Trimper, J.B., Hu, C., Levey, A.I., Manns, J.R., Effects of selective M1Muscarinic receptor activation on hippocampal spatial representations and neuronal oscillations. ACS Chem. Neurosci. 2016, 7, 1393-1405. https://doi.org/10.1021/ acschemneuro.6b00160.

24. Stoiljkovic, M., Leventhal, L., Chen, A., Chen, T., Driscoll, R., Flood, D., Hodgdon, H., Hurst, R., Nagy, D., Piser, T., Tang, C., Townsend, M., Tu, Z., Bertrand, D., Koenig, G., Hajos, ' M., Concentrationresponse relationship of the $\alpha 7$ nicotinic acetylcholine receptor agonist FRM-17874 across multiple in vitro and in vivo assays. Biochem. Pharmacol. 2015, 97, 576-589. https://doi.org/10.1016/j. bcp.2015.07.006.

25. Ziyatdinova, S., Gurevicius, K., Kutchiashvili, N., Bolkvadze, T., Nissinen, J., Tanila, H., Pitk" anen, A., Spontaneous epileptiform discharges in a mouse model of Alzheimer's disease are suppressed by antiepileptic drugs that block sodium channels. Epilepsy Res. 2011, 94, 75-85. https://doi.org/10.1016/j.eplepsyres.2011. 01.003 .
26. Sanchez-Rodríguez,' I., Temprano-Carazo, S., $\mathrm{N}^{\prime}$ ajera, A., Djebari, S., Yajeya, J., Gruart, A., Delgado-García, J.M., Jim’enezDíaz, L., Navarro-Lopez, ' J.D., Activation of G-protein-gated inwardly rectifying potassium (Kir3/GirK) channels rescues hippocampal functions in a mouse model of early amyloid- $\beta$ pathology. Sci. Rep. 2017, 7, 14658. https://doi.org/10.1038/s41598017-15306-8.

27. Li, C., Liu, W., Li, X., Zhang, Z., Qi, H., Liu, S., Yan, N., Xing, Y., Holscher, " C., Wang, Z., The novel GLP-1/GIP analogue DA5-CH reduces tau phosphorylation and normalizes theta rhythm in the ICV. STZ rat model of AD. Brain Behav. 10, 2020, https:// doi.org/10.1002/brb3.1505.

28. Ansari, N.; Khodagholi, F. Natural products as promising drug candidates for the treatment of Alzheimer's disease: Molecular mechanism aspect. Curr. Neuropharmacol. 2013, 11, 414-429.

29. D’Onofrio, G.; Sancarlo, D.; Ruan, Q.; Yu, Z.; Panza, F.; Daniele, A.; Greco, A.; Seripa, D. Phytochemicals in the Treatment of Alzheimer's Disease: A Systematic Review. Curr. Drug Targets 2017, 18, 14871498.

30. Sanka N, Santhipriya N and Nadendla RR. An updated review on Anti-Alzheimer's herbal drugs. Journal of Drug Delivery and Therapeutics 2018; 8(6):360-372.

31. Mirjalili MH, Moyano E, Bonfill M, Cusido RM, Palazón J. Steroidal Lactones from Withania somnifera, an Ancient Plant for Novel Medicine. Molecules 2009;14:23732393.

32. Choudhary D, Bhattacharyya S, Bose SE. cacy and Safety of Ashwagandha (Withania somnifera (L.) Dunal) Root Extract in Improving Memory and Cognitive Functions. J. Diet. Suppl 2017; 14:599-612.

33. Chaudhari KS, Tiwari NR, Tiwari RR, Sharma RS. Neurocognitive Effect of Nootropic Drug Brahmi (Bacopa monnieri) in Alzheimer's Disease. Ann. Neurosci 2017;24: 111-122.

34. Snow AD, Castillo GM, Nguyen BP, Choi PY, Cummings JA, Cam J et al. The Amazon rain forest plant Uncaria tomentosa (cat's claw) and its specific proanthocyanidin constituents are potent inhibitors and reducers of both brain plaques and tangles. Sci. Rep 2019;9:561 
35. Bastianetto S, Zheng WH, Quirion R. The Ginkgo biloba extract (EGb 761) protects and rescues hippocampal cells against nitric oxide-induced toxicity: Involvement of its flavonoid constituents and protein kinase $\mathrm{C}$. J. Neurochem 2000; 74:2268-2277.

36. Parihar MS, Hemnani, T. Phenolic antioxidants attenuate hippocampal neuronal cell damage against kainic acid induced excitotoxicity. J. Biosci 2003; 28:121-128.

37. John M, Ringman, Jeffrey L, Cummings Sally, A Frautschy, Gregory $\mathrm{M}$ et al. Masterman. A Potential Role of the Curry Spice Curcumin in Alzheimer's disease. Curr Alzheimer Res 2005; 2(2):131-136.

38. Bihaqi SW, Sharma M, Singh AP, T iwari M: Neuroprotective role of Convolvulus pluricaulis on aluminium induced neurotoxicity in rat brain. J Ethnopharmacol 2009;124:409-415.

39. Gorji, N.; Moeini, R.; Memariani, Z. Almond, hazelnut and walnut, three nuts for neuroprotection in Alzheimer's disease: A neuropharmacological review of their bioactive constituents. Pharm. Res. 2018, 129, 115-127.

40. Chauhan, A.; Chauhan, V. Beneficial Effects of Walnuts on Cognition and Brain Health. Nutrients 2020, 12, 550.

41. Martins, M.; Silva, R.; Pinto, M.M.M.; Sousa, E. Marine Natural Products, Multitarget Therapy and Repurposed Agents in Alzheimer's Disease. Pharmaceuticals 2020, 13, 242.

42. Zhu, B.; Li, Z.; Qian, P.Y.; Herrup, K. Marine bacterial extracts as a new rich source of drugs against Alzheimer's disease. J. Neurochem. 2020, 152, 493-508.

How to cite this article: A.Mary Prashanthi, Lakshmi VN. Pharmacological and neuroprotective natural products in the treatment of Alzheimer's Disease. International Journal of Science \& Healthcare Research. 2021; 6(3): 366-382. DOI: https://doi.org/10.52403/ijshr. 20210762 